\title{
The Scientific Need for a Dedicated Interplanetary Dust Instrument at Mars
}

Fries, M. ${ }^{1}$, Ashley, J. ${ }^{2}$, Beegle, L. ${ }^{2}$, Bhartia, R., Bland, P. ${ }^{3}$, Burton, A. ${ }^{1}$, Butterworth, A.L. ${ }^{4}$, Cooke, W. ${ }^{5}$, Conrad, P. ${ }^{6}$, Christou, A. ${ }^{7}$, Crismani, M. ${ }^{8}$, Engrand, C. ${ }^{9}$, Dartois, E. ${ }^{10}$, Duprat J. ${ }^{11}$, Flynn, G. ${ }^{12}$, Fisher, K. ${ }^{1}$, Gainsforth, Z. ${ }^{4}$, Genge, M. ${ }^{13}$, Graham, L. ${ }^{1}$, Horanyi, M. ${ }^{14}$, Janches, D. ${ }^{15}$, ten Kate, I.L. ${ }^{16}$, New, J.S. ${ }^{4}$, Plane, J. ${ }^{17}$, Rojas, J. ${ }^{9}$, Sephton, M. ${ }^{13}$, Steele, A. ${ }^{6}$, Sykes, M. ${ }^{18}$, Welzenbach, L. ${ }^{19}$, Zolensky, M. ${ }^{1}$

${ }^{1}$ NASA Astromaterials Research and Exploration Science (ARES), Johnson Space Center, Houston, TX 77058, (281) 244-1455, marc.d.fries@nasa.gov

${ }^{2}$ Jet Propulsion Laboratory, California Institute of Technology, Pasadena, CA 91109 ${ }^{3}$ Space Science and Technology Centre, Curtin University, Perth, Australia, WA 6845 ${ }^{4}$ Space Sciences Laboratory, University of California - Berkeley, Berkeley, CA 94720 ${ }^{5}$ NASA Meteoroid Environment Office, Marshall Space Flight Center, Huntsville, AL 35812

${ }^{6}$ Geophysical Laboratory, Carnegie Institute for Science, Washington DC 20015 ${ }^{7}$ Armagh Observatory, Armagh BT61 9DG, U.K.

${ }^{8}$ NPP/USRA, NASA Goddard Space Flight Center, Planetary Systems Laboratory, Code 693, Greenbelt, MD, USA

'Université Paris-Saclay, CNRS, IJCLab, Bâtiment 104, 91405 Orsay Campus, FR.

${ }^{10}$ Institut des Sciences Moléculaires d'Orsay, UMR8214, CNRS, Université Paris-Saclay, 91405 Orsay, France

${ }^{11}$ IMPMC, CNRS-MNHN-Sorbonne Université, 57 rue Cuvier, 75005 Paris, FR.

${ }^{12}$ State University of New York (SUNY) Plattsburgh, Plattsburgh, NY 12901

${ }^{13}$ Imperial College London, London U.K. SW7 2AZ

${ }^{14} \mathrm{CU} / \mathrm{LASP}$, Boulder, Colorado 80303

${ }^{15}$ NASA Goddard Space Flight Center, Greenbelt, MD 20771

${ }^{16}$ Utrecht University, Princetonlaan 8A, 3584 CB Utrecht, The Netherlands

${ }^{17}$ University of Leeds, Woodhouse, Leeds LS2 9JT, U.K.

${ }^{18}$ Planetary Science Institute, Tucson, AZ 85719

${ }^{19}$ Open University, Milton Keynes MK7 6AA, UK

Signatories:

Devanshu, J., MVJ College of Engineering,Whitefield,Bangalore, India

Contact the corresponding author for a list of references: marc.d.fries@nasa.gov 


\section{ABSTRACT}

Interplanetary dust is a scientifically important constituent of the Solar System that consists of material shed by asteroids, comets, and other airless bodies. As used here, the term "dust" includes interplanetary dust particles and micrometeoroids. Dust has been studied by missions such as Mariner, Pioneer, and Voyager in both interplanetary space and in the vicinity of most of the planets.To date, however, no dedicated interplanetary dust instrument has yet been employed for detailed analysis of the dust environment of Mars. Partial data on dust flux has been provided by the 1965 Mariner IV flyby, the MAVEN orbiter, and other missions, but a complete understanding of interplanetary dust abundance, composition, debris hazard, annual flux variation, and origins is lacking. These data are critical for understanding the effects of dust upon the martian system, including the carbonaceous input into the regolith of Mars and its moons, the chemical input into the martian atmosphere, potential effects upon remote sensing data, the hypothesized existence of a Phobos dust ring, and possible annual variations from meteor shower infall. These effects have direct ramifications for interpretation of Mars/Phobos/Deimos mission science and analysis of returned samples from those worlds. To remediate this shortfall, the authors recommend that a dedicated interplanetary dust analysis instrument should be included in the instrument package for an upcoming martian orbiter in the near term. Such an interplanetary dust analysis instrument should collect data over a time period of several martian years in order to generate a statistically robust data set on interplanetary dust concentration and flux over a wide range of mass, and to discern temporal variation over multiple martian years.

\section{BACKGROUND}

\section{The Science of Dust Measurements}

Interplanetary dust imposes a variety of scientifically important effects on planetary bodies. These include material input into atmospheres (Ferguson and Fehsenfeld 1968, Plane 2012, Crismani et al 2017, Grebowsky et al 2017), delivery of carbonaceous and volatile compounds of prebiotic and astrobiological importance (Flynn 1996), addition of significant amounts of exogenous material to planetary and small-body regolith (Clark and Baird 1979, Moores and Schuerger 2012), modification of remote sensing data (Flynn 1991, Fries et al., 2016, Plane et al., 2018), and variable changes to the exospheres of airless bodies (Cremonese et al., 2005; Pokorny et al., 2018; Janches et al., 2018, Pokorny et al., 2019). Additionally, dust constitutes a hazard to spacecraft and must be characterized prior to arrival of humans in martian orbit (Moorhead et al., 2020). Interplanetary dust has long been considered an important 
constituent of Solar System materials as evidenced by the large number of spaceflight instruments dedicated to their analysis, covering the environments of the Earth and Moon, Venus, Jupiter, Saturn, Pluto, small bodies (comets and asteroids) and interplanetary space. The JAXA Nozomi probe included the Mars Dust Counter (MDC), in recognition of the scientific importance of understanding dust in the martian system, but that spacecraft unfortunately did not achieve orbit around Mars (Sasaki et al., 2002).

Interplanetary dust is derived from airless bodies, principally asteroids and comets. On planetary bodies, dust infall occurs from two major sources: sporadic infall and meteor showers. Sporadic infall arises from a mixed contribution of particles from asteroids and comets. In the Solar System, individual particles are dynamically scattered to the point that their orbits are no longer indicative of their parent body (Nesvorny et al., 2010, Nesvorny et al., 2011a, Nesvorny et al., 2011b, Pokorny et al., 2014). Meteor showers are short-lived events arising from material ejected from its parent body entrained in the orbital path of a comet or other dust-generating body, and the showers manifest themselves as short-lived infall from a well-defined vector (Jenniskens, 2006). These particles are fragments of the originating body and their orbits are indicative of the parent body or at least of the parent body at the time of ejection of the dust (Jenniskens, 2006; Janches et al., 2020). Sporadic infall comprises the majority of the total annual infall mass, although meteor showers can exceed the sporadic infall rate over the timespan of the meteor shower. While meteor showers on Mars must exist, they have not been identified to date. An interplanetary dust analysis ("IDA" hereafter for brevity) instrument may remedy that shortcoming.

\section{Hazards to Both Crewed and Uncrewed Spacecraft}

Hazards from interplanetary dust impacts are a concern for both uncrewed and crewed spacecraft, and the extent of these hazards have not yet been adequately described for cis-martian space. NASA has documented at least two instances of significant damage to interplanetary spacecraft to date. One was Mariner 2's sudden loss of attitude control on 08 Sep 1962 possibly caused by collision with a natural object (NSSDCA 2020a). Another was Mariner 4's encounter with a debris stream on 15 Sep 1967 which physically slewed the spacecraft and damaged its thermal management system (NSSDC 2020b). With this in mind, NASA's Human Exploration Operations and Mission Directorate (HEOMD) has established debris-specific Strategic Knowledge Gaps (SKGs) that must be addressed prior to crewed flights to cis-martian space. HEOMD identifies impact hazards with SKG A.3 "Orbital particulates", stating "[w]e have insufficient information about the orbital particulate environment in high-Mars orbit that may impact the delivery of cargo and crew to the Martian system." For crewed missions to Phobos and Deimos, debris impact hazards are listed as SKG B.2.2. The "Gap Filling Activity" intended to resolve these SKGs is a dust analysis measurement in martian 
orbit, in agreement with the intent of this white paper (MEPAG 2012). These SKGs could be resolved with a dedicated IDA instrument in martian orbit.

\section{Current Knowledge of the Dust Environment of the Martian System}

Dust comprises a significant infall on Mars and has important effects upon martian atmospheric and surface science, but those effects - to include infall mass and temporal variation - are inadequately understood at present. On Earth, direct measurements show that dust dominates the total annual infall mass. Impacts on the Long Duration Exposure Facility (LDEF) indicate the current infall rate of dust is $110 \pm 55$ tonnes/day (Flynn and McKay 1990, Flynn 1996, Love and Brownlee 1993, Plane et al., 2018 ) and that this infall rate is $\sim 100 x$ the mass of annual mean meteorite infall (Bland et al., 1996). Translating terrestrial infall to Mars, estimates of the amount of accumulated infall in martian regolith range from 1-3\% (Yen et al., 2006), up to $22 \%$ (Morris et al., 2000), and 2-29\% (Flynn and McKay 1990). Yen et al. (2006) found this component in both regolith and some sedimentary rocks. Given the wide range of estimated values there is room for improvement in this measurement, and that improvement would be granted by an IDA instrument.

Refinement of the martian infall rate would yield direct benefits to landed mission science as well. Coupled with refined measurement of the dust flux at Mars, the concentration of infall in sedimentary rocks would provide a measure of the sedimentation rate, a well-established technique applied to terrestrial oceanic sediments. Similarly, the flux measurement combined with a determination of its concentration in the regolith can be used to determine the regolith production rate (Flynn and McKay, 1990).

Much of the dust flux is related to carbonaceous chondrites (with a few wt $\%$ of C), however, a minor part of the particles (i.e. ultra-carbonaceous micrometeorites) exhibit much larger carbon concentration (Duprat et al. 2010) and average carbon content of dust on Earth has been estimated at $12 \mathrm{wt. \%}$ (Thomas et al., 1993). Nevertheless, carbon deposited as a component of dust is an insignificant contributor to the total carbon budget of the Earth's surface, that already contains $>45$ gigatonnes of carbon (excluding the lithosphere, Falkowski et al 2000). On Mars, however, carbon from infall may be the greatest flux of carbon onto the martian surface. Mars' smaller size, lower mean infall velocity, and oxidized atmosphere allow significantly more dust (and carbon) to reach the surface unaltered, per unit of infall. Flynn (1996) estimates that an order of magnitude more carbon per unit area has been delivered to Mars' surface than Earth's over geologic time. Mars lacks Earth's vast oceans, active volcanism, and crustal recycling, and so the planet is without several of Earth's major carbon sinks and fluxes. Carbon infall on Mars is believed to be an important factor in landed mission science. Accumulated carbon has been estimated to amount to $\sim 500$ 
ppm of oxidized carbon in the form of benzenecarboxylic acid (Benner et al 2000), and Carillo-Sanchez et al (2020) estimated $\sim 10$ ppm carbon from infall has accumulated in the top meter of regolith. Moores and Shuerger (2012) estimated that carbon from dust would accumulate to 3.4 and $4.9 \mathrm{ppm}$ at the Viking 1 and 2 landing sites, respectively. Considerable uncertainty exists in the chemical degradation of carbon compounds such that lifetime and accumulation calculations vary, demonstrating the need to better constrain annual infall, which could be achieved with a dedicated IDA instrument at Mars.

\begin{tabular}{|c|c|c|c|c|}
\hline Spacecraft & Distance From Sun (AU) & Detector Area $\left(\mathrm{m}^{2}\right)$ & Mass Sensitivity (g) & Reference \\
\hline \multicolumn{5}{|c|}{ Dedicated Dust Analysis Instruments } \\
\hline Cassini & $0.72-10$ & 0.1 & $10^{-16}-10^{-6}$ & Srama et al (2004) \\
\hline Galileo & $0.7-5.4$ & 0.1 & $>10^{-19}-10^{-16}$ & Grün et al (1992a) \\
\hline HEOS 2 & 1 & 0.01 & 0.01 & Dietzel et al (1973) \\
\hline Helios 1/2 & $0.3-1$ & 0.012 & $>10^{-17}-10^{-13}$ & Dietzel et al (1973) \\
\hline LADEE & 1 & 0.01 & $>10^{-12}$ & Horányi et al (2014) \\
\hline Mariner IV (Mars flyby) & $1-1.56$ & 0.35 & $10^{-11}-10^{-9}$ & Alexander et al (1967) \\
\hline New Horizons & $1-48 * *$ & 0.1 & $>10^{-12}$ & Horányi et al (2018) \\
\hline Nozomi & $1-1.5$ & 0.014 & $10^{-15}-10^{-10}$ & Sasaki et al (2001) \\
\hline Pioneer 8 & $0.75-1.09$ & 0.0094 & $>10^{-13}$ & Berg \& Richardson (1969) \\
\hline Pioneer 9 & $0.75-0.99$ & 0.0074 & $>10^{-13}$ & Rhee et al (1974) \\
\hline Pioneer 10 & $1-66$ & $0.26 *$ & $>10^{-9}-10^{-8}$ & Humes (1980) \\
\hline Pioneer 11 & $1-47$ & $0.56^{*}$ & $>10^{-9}-10^{-8}$ & Humes (1980) \\
\hline Ulysses & $1-5.4$ & 0.1 & $>10^{-19}-10^{-16}$ & Grün et al (1992b) \\
\hline \multicolumn{5}{|c|}{ Opportunity Analyses Using Other Instruments } \\
\hline Voyager 1 Plasma Wave Inst. & $1-148$ & 1.66 & $>10^{-9}$ & Gurnett et al (1997) \\
\hline Voyager 2 Plasma Wave Inst. & $1-122$ & 1.66 & $>10^{-9}$ & Gurnett et al (1997) \\
\hline MAVEN Langmuir Probe & 1.5 & $\sim 9$ & $\sim 10^{-9}-10^{-6}$ to $10^{-11}-10^{-9}$ & Andersson et al (2015) \\
\hline MAVEN IUVS & 1.5 & N/A & (Detection of ions) & Crismani et al (2017) \\
\hline \multicolumn{5}{|c|}{ *Initial area, actual area decreased as the pressurized cells were punctured } \\
\hline ** As of $6 / 2020$ & & & & \\
\hline
\end{tabular}

Table 1: Previous dust detection measurements by the locale and sensitivity parameters of their measurements. Missions highlighted in yellow include measurements made at Mars' orbit but not near Mars itself. Mariner 4 (green highlight) was a Mars flyby mission which measured dust flux near Mars during its passage. MAVEN (red highlight) made some measurements from martian orbit but did not include a dedicated dust instrument. MAVEN's Langmuir probe detected impacts via transient voltage spikes, sufficient to constrain the number of impacts but without data on composition, mass, or origin. MAVEN (IUS) also detected Mg+ ion concentration from infall in Mars' upper atmosphere. While total flux has been estimated from multiple missions, to date the composition, origin, annual variation, and velocity distribution have not been measured for infall onto Mars. 
It has also been hypothesized that Mars features a diffuse dusty ring at Phobos' orbit, that, if true, would constitute the only known ring around a terrestrial planet (Soter (1971), Sasaki (1999)). One of the main mission goals of the Mars Dust Counter on the JAXA Nozomi spacecraft was to investigate this possibility, but Nozomi did not achieve orbit around Mars. The hypothesis of a martian dust ring remains untested but could be explored by a dedicated IDA instrument.

Infall flux contributes material to the martian surface that directly affects landed missions and must be considered for Mars sample return, both during sample selection and in the course of analyses of the returned samples themselves. Of special interest is the fact that carbonaceous material in interplanetary dust can make up over $90 \%$ of individual grains by volume, with weight-percent levels of carbonyls and aliphatic compounds (Thomas et al., 1993, Flynn et al., 2003, Duprat et al., 2010). As a result, incomplete constraints on the composition and concentration of infall may obfuscate attempts to search for past life on Mars and may complicate analyses of returned martian samples. For Phobos and Deimos, Mars' gravity drives the moons' carbon infall flux, and the resulting accumulation of carbonaceous infall has been proposed as an explanation for the moons' modern reflectance spectra (Flynn 1991, Fries et al., 2016, Plane et al., 2018). A complete understanding of the flux, composition, and accumulation processes obtainable with a dedicated IDA instrument will be necessary in order to constrain modern concentrations on Mars and the martian moons.

\section{Martian Interplanetary Dust Measurements to Date}

Overall, dust input onto the martian surface is a significant factor for a robust interpretation of landed mission data, but infall flux, accumulation, and composition are insufficiently described at present. While modeled values for flux exist (Flynn 1996, Carillo-Sanchez et al., 2018, Plane et al., 2018, Carillo-Sanchez et al., 2020) as well as limited measurements from the Mariner 4 flyby (Alexander et al 1967) and MAVEN missions (Crismani et al., 2017), scientifically important uncertainties regarding the flux, annual variations, composition, and origin of martian dust infall persist. Inclusion of a dedicated dust IDA instrument on an upcoming martian orbiter can remedy the need to clarify this uncertainty.

Estimates of martian infall flux have varied considerably as methods and observations have evolved. Flynn (1996) estimated an annual flux of 8,600 tonnes/year over the mass range of $10^{-1}$ to $10^{-15} \mathrm{~g}$ on the basis of comparison with measured values for Earth. Andersson et al (2015) estimated 31.5 - 3,150 tonnes/year based on impacts measured by the MAVEN Langmuir Probe. Crismani et al (2015) estimated 1,374-2,061 tonnes/year based on MAVEN measurements of $\mathrm{Mg}+$ ions in the martian upper atmosphere. Carillo-Sanchez et al (2020) recently estimated an annual infall mass of 329-1,205 tonnes/year based on dynamical modeling of dust from asteroidal and 
cometary sources. Flynn et al (1996) and Carillo-Sanchez et al (2020) both indicate that survival of carbonaceous material should be much higher for Mars than for Earth due to lower infall velocity, possibly leading to significant concentrations on the planet's surface. Annual variations in infall flux due to meteor shower activity are poorly understood. One attempt has been made to observe meteors directly using a camera on a Mars Exploration Rover (MER) but it was effectively obscured by radiation-induced noise in the camera (Domokos et al 2007). The impact dataset reported for MAVEN (Andersson et al 2015) may contain a record of annual variation but this has not been explored. Crismani et al (2015) reported non-detection of meteor shower activity in upper-altitude based on observed $\mathrm{Mg}+$ concentration. To date, no measurements have been published showing annual activity of martian meteor showers even though they are widely believed to exist (Christou and Buerle 1999, Treiman and Treiman 2000, Christou 2005, Christou 2010). This hole in our understanding could be closed by direct measurement of dust with a dedicated IDA instrument.

\section{SUMMARY}

A significant disparity exists between the scientific importance of dust flux at Mars and measurement assets that have been deployed there to date. One mission (Nozomi) recognized the scientific need to resolve this discrepancy and included a dust characterization IDA instrument, but that mission unfortunately did not achieve martian orbit. Dust infall has significant effects on safety for both robotic and crewed spaceflight, on total carbon infall onto the surfaces of Mars and its moons, on extraterrestrial infall into materials sampled and analyzed by landed missions, on the composition of the martian atmosphere, and on spectroscopic measurements of the planet and its moons. Measurements have been made on previous missions but significant improvements are needed in terms of direct measurement of flux, composition, origin, and velocity and mass distributions. We recommend that NASA address these deficiencies with a dedicated dust analysis IDA instrument in a near-term future Mars orbiter mission.

No specific instrument is recommended here, but multiple instrument options are reasonably expected to be available based on IDA instruments flown on recent missions. With due consideration for mass, power, cost, operations, and other typical space flight constraints, we recommend the following:

- NASA should include a dedicated interplanetary dust analysis instrument on an upcoming Mars orbiter mission, either flown or at least developed within the next decade.

- It is scientifically important to measure:

- Infall flux over a wide range of particle sizes. 
- Infall originating direction over a sufficiently broad area of the sky such that sources of input and originating orbits can be discerned.

- Infall composition especially in elements which will affect regolith composition in the martian system, e.g. C, H, N, P, Ni.

- Infall velocity to determine the total amount of carbon that survives infall to reach the martian surface.

- Infall annual flux variation to identify the sources, flux, and mass contribution of meteor showers on Mars.

- The IDA instrument should collect data for multiple martian years to achieve statistically robust results and to reliably discern annual flux variation.

Text in orange indicates measurements that have been made either indirectly or while passing through Mars' orbit. Text in red indicates measurements that have not been made to date (see Figure 1).

\section{Definition of "Interplanetary Dust" Used Here}

The scientific concerns addressed in this white paper focus on the dust and meteoroid environments of interplanetary origin around Mars, and we will use the International Astronomical Union (IAU) Commission F1: Meteors, Meteoroids and Interplanetary Dust definition of terms approved in 2016. To wit, a "meteoroid" is a solid natural object of a size roughly between 30 micrometers and 1 meter. Dust (interplanetary) is finely divided solid matter, with particle sizes in general smaller than meteoroids, moving in, or coming from, interplanetary space (i.e. space within our Solar System). Even though some overlap exists between "dust" and "meteoroid" we use dust as a catchall term for brevity. Smaller examples (typically 10-100 microns in size) have been collected in the atmosphere and are (historically) called interplanetary dust particles (IDPs). Larger examples (typically between $\sim 30 \mu \mathrm{m}$ and $\approx 500$ microns in size) can be collected on the ground and are called micrometeorites. When in interplanetary space, they are collectively called dust particles. While small dust particles do not give rise to meteor phenomena visible to the naked eye, meteoroids can do so. While all infalling particles experience some degree of heating, a fraction of these particles are heated below their evaporation point and settle from the high atmosphere to the ground at time scales depending on their sizes with larger particles sedimenting faster. These unmelted particles can still hold signatures from their atmospheric entry (e.g. magnetite rims, vesicles, loss of volatile elements, chemical alteration).

References: Contact the corresponding author for a list of references (marc.d.fries@nasa.gov). 